\title{
SLOVAK DEPENDENCY TREEBANK IN UNIVERSAL DEPENDENCIES
}

\author{
DANIEL ZEMAN \\ Faculty of Mathematics and Physics, Charles University, Prague, Czech Republic \\ ZEMAN, Daniel: Slovak Dependency Treebank in Universal Dependencies. Journal \\ of Linguistics, 2017, Vol. 68, No 2, pp. $385-395$.
}

\begin{abstract}
We describe a conversion of the syntactically annotated part of the Slovak National Corpus into the annotation scheme known as Universal Dependencies. Only a small subset of the data has been converted so far; yet it is the first Slovak treebank that is publicly available for research. We list a number of research projects in which the dataset has been used so far, including the first parsing results.

Keywords: treebank, dependency, universal dependencies, syntax, morphology, tagging, parsing
\end{abstract}

\section{INTRODUCTION}

Syntactically annotated corpora (treebanks) are important language resources, indispensable for linguistic research and natural language processing alike. Modern treebanks are mostly built on the notion of dependency relations. With the increasing number of languages covered and amount of data available, there is a growing interest in finding one common, linguistically adequate and cross-linguistically applicable annotation style $[5,13]$. Universal Dependencies (UD) ${ }^{1}[6]$ is an international effort aimed at such an annotation standard; at the same time, UD also releases treebanks annotated according to the UD guidelines, and has arguably become the largest collection of freely available dependency treebanks worldwide.

UD treebanks are released twice a year and every release so far added several languages that had not been part of the previous releases. The group of Slavic languages is represented quite well. [12] gave an early account of Slavic languages in UD 1.1, as well as an overview of other Slavic treebanks outside UD (Table 1). At the time of this writing, UD 2.0 is the most recent release and it comprises 70 treebanks of 50 languages; among them, nearly all ${ }^{2}$ Slavic languages are represented with at least a small dataset (Table 2).

In the present article we focus on one of the recent additions to UD, the Slovak Dependency Treebank.

${ }^{1}$ http: //universaldependencies.org/

${ }^{2}$ Serbian and Upper Sorbian are ready to be released in UD 2.1. What remains missing is Lower Sorbian, Bosnian/Montenegrin and Macedonian; and one may also argue for some smaller languages with less clear status such as Kashubian or Rusyn. 


\begin{tabular}{|l|l|l|l|l|}
\hline Language & Code & Treebank & Sent & Tok \\
\hline Bulgarian & {$[\mathrm{bg}]$} & BulTreeBank & 13,221 & $196 \mathrm{~K}$ \\
\hline Church Slavonic & {$[\mathrm{cu}]$} & PROIEL & 7,818 & $72 \mathrm{~K}$ \\
\hline Croatian & {$[\mathrm{hr}]$} & SETimes.HR & 3,736 & $84 \mathrm{~K}$ \\
\hline Czech & {$[\mathrm{cs}]$} & PDT & 87,913 & $1504 \mathrm{~K}$ \\
\hline Polish & {$[\mathrm{pl}]$} & IPI PAN & 8,227 & $84 \mathrm{~K}$ \\
\hline Russian & {$[\mathrm{ru}]$} & SynTagRus & 63,000 & $900 \mathrm{~K}$ \\
\hline Slovak & {$[\mathrm{sl}]$} & SNK & 63,238 & $994 \mathrm{~K}$ \\
\hline Slovenian & {$[\mathrm{sl}]$} & SSJ500K & 27,829 & $500 \mathrm{~K}$ \\
\hline
\end{tabular}

Tab. 1. Dependency treebanks of Slavic languages, as listed by [12] (only some of them were converted to UD at that time)

\section{SLOVAK DEPENDENCY TREEBANK}

The data in the Slovak treebank come from the Slovak National Corpus (Slovensky národný korpus, SNK) ${ }^{3}$ [8]. Over 63,000 sentences (almost one million words) received manual morphological and syntactic annotation, making it one of the three largest treebanks of Slavic languages (after the Czech PDT [1], ${ }^{4}$ and with similar size to the Russian SynTagRus [2]). [8] describe the composition of the treebank as $78 \%$ fiction, $13 \%$ scientific and 9\% journalistic text. An important point is that it includes free sources like Wikipedia or folk tales, where intellectual property rights do not complicate access to and distribution of the annotated data. Most sentences were independently annotated by two annotators in order to identify difficult phenomena and reduce annotation errors. The positions where the two annotators disagree would eventually be decided by a third annotator. Unfortunately, this final step has not been completed for all the sentences, which also means that the treebank has yet to wait for its full official release. ${ }^{5}$

\begin{tabular}{|l|l|l|l|l|}
\hline Language & Code & Treebank & Sent & Tok \\
\hline Belarusian & {$[\mathrm{be}]$} & UD & 393 & $8 \mathrm{~K}$ \\
\hline Bulgarian & {$[\mathrm{bg}]$} & BulTreeBank & 11,138 & $156 \mathrm{~K}$ \\
\hline Church Slavonic & {$[\mathrm{cu}]$} & PROIEL & 6,337 & $58 \mathrm{~K}$ \\
\hline Croatian & {$[\mathrm{hr}]$} & SETimes.HR & 8,889 & $197 \mathrm{~K}$ \\
\hline Czech & {$[\mathrm{cs}]$} & PDT & 87,913 & $1506 \mathrm{~K}$ \\
\hline Czech & {$[\mathrm{cs}]$} & CAC & 24,709 & $494 \mathrm{~K}$ \\
\hline Czech & {$[\mathrm{cs}]$} & CLTT & 1,125 & $38 \mathrm{~K}$ \\
\hline Czech & {$[\mathrm{cs}]$} & PUD & 1,000 & $19 \mathrm{~K}$ \\
\hline Polish & {$[\mathrm{pl}]$} & IPI PAN & 8,227 & $84 \mathrm{~K}$ \\
\hline Russian & {$[\mathrm{ru}]$} & Google & 5,030 & $99 \mathrm{~K}$ \\
\hline Russian & {$[\mathrm{ru}]$} & SynTagRus & 61,889 & $1107 \mathrm{~K}$ \\
\hline
\end{tabular}

${ }^{3}$ http://korpus.juls.savba.sk/

${ }^{4}$ http://ufal.mff.cuni.cz/pdt

${ }^{5}$ The Slovak Language Treebank has been listed in META-SHARE since 2011 (http:// metashare.korpus.sk/repository/browse/slovak-treebank/36e46d0a649311e2

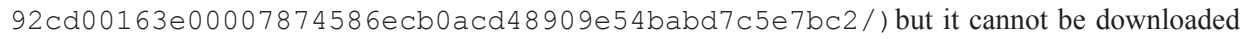
from there. 


\begin{tabular}{|l|l|l|l|l|}
\hline Russian & {$[\mathrm{ru}]$} & PUD & 1,000 & $19 \mathrm{~K}$ \\
\hline Serbian* & {$[\mathrm{sr}]$} & SETimes.SR & 3,891 & $87 \mathrm{~K}$ \\
\hline Slovak & {$[\mathrm{sl}]$} & SNK & 10,604 & $106 \mathrm{~K}$ \\
\hline Slovenian & {$[\mathrm{sl}]$} & SSJ200K & 8,000 & $141 \mathrm{~K}$ \\
\hline Slovenian & {$[\mathrm{sl}]$} & SST & 3,188 & $29 \mathrm{~K}$ \\
\hline Ukrainian & {$[\mathrm{uk}]$} & UD & 1,706 & $26 \mathrm{~K}$ \\
\hline Upper Sorbian & {$[\mathrm{hsb}]$} & UD & 646 & $11 \mathrm{~K}$ \\
\hline
\end{tabular}

Tab. 2. Slavic treebanks in UD release 2.0 (plus Serbian, scheduled for UD 2.1). Note that there were two UD 2.0 releases and the counts in this table sum up both: First, training and development data were released in March 2017. The test sets were kept aside for the CoNLL 2017 Shared Task in dependency parsing, and they were released in May 2017 after the shared task.

Morphological annotation in SNK assigns to each word (token) its lemma and a morphological tag that encodes its part of speech and values of relevant morphological features: inflection type, gender, number, case, degree of comparison, agglutination (preposition + pronoun), verbal form, aspect, polarity, voice etc. ${ }^{6}$

The syntactic annotation follows the annotation guidelines of the "analytical layer" of the Prague Dependency Treebank. ${ }^{7}$

The following steps have been taken to ensure quality of the data. Note that these are filtering steps - on the first sign of a problem, the entire unit (sentence or file) is discarded. In most cases it should be possible to manually fix the problem and retain the sentence; however, the obvious short-term advantage of the filtering approach is that it requires fewer human resources and the problem-free part of the data can be made available sooner.

- $\quad$ Removed files where the morphological annotation was not manual.

- $\quad$ Removed files where the syntactic annotation was done only by one annotator.

- Removed files where the annotators disagree in sentence segmentation (different number of sentences).

- $\quad$ Removed sentences where the annotators disagree in tokenization (different number of tokens).

- Removed empty sentences and sentences with just one token.

- Removed sentences where one or more annotation items (lemmas, morphological tags, dependency relation labels) were empty.

The resulting corpus consisted of 40,350 sentences and 671,968 tokens. Every sentence in this data set had two complete dependency trees from two annotators. In general, the contrasted annotations were not expected to differ on the word level and in morphological annotation because the annotators were focusing on syntax (while morphology was inherited from pre-existing annotation of SNK). However, it seems that they occasionally modified the lower layers: there were 747 mismatches in word forms, 2 in lemmas and 3 in morphological tags. Again, all affected sentences were removed.

${ }^{6}$ See http://korpus.juls.savba.sk/attachments/morpho/tagset-www.pdf for documentation of the tagset (in Slovak).

7 See http://ufal.mff.cuni.cz/pdt2.0/doc/manuals/en/a-layer/html/ index.html for documentation. 
As for the syntactic annotation, the two annotators agreed on $80.34 \%$ dependencies (both the parent node index and the dependency label). If we disregard the dependency labels and only look at the parent node assignment, the agreement rate rises to $87.31 \%$. It means that in $6.97 \%$ of all tokens ( $35 \%$ of dependency errors) the sole disagreement is in the label (termed analytical function or afun in Praguestyle treebanks).

Finally, let us consider "complete matches" - sentences whose dependency trees from the two annotators were identical in all aspects of annotation. These sentences constitute the most trustworthy core of the corpus, as it is unlikely that two annotators independently make the same error. Only completely matching sentences were selected for the first UD release of the treebank: reliability of the annotation got the top priority. Of course, there are also some downsides to this decision. The first of them is linked to filtering in general: the resulting corpus does not contain whole documents, making any discourse-level studies impossible. The second drawback is perhaps even more serious: the treebank contains a high proportion of short sentences because the more words in the sentence, the higher is the probability of an annotation error. Before removing sentences with annotation mismatches, the average sentence length in the treebank was 16.7 tokens. When only complete matches remained, the average length dropped to 10.0 tokens. (The longest completely matched sentence contained 54 tokens.) Such a corpus is unbalanced and some more complex grammatical structures may be seriously underrepresented in it. It is thus highly desirable to extend the corpus and add more sentences in the future. However, the filtered portion is arguably much better than nothing, and can be used to train statistical parsers for Slovak; with 10,604 sentences and 106,043 tokens it is still a medium-sized treebank, surpassing by an order of magnitude treebanks that are available for some other languages.

Given that there was no official download site for the Slovak treebank, the filtered part was first released, with the permission from the L'udovít Štúr Institute of Linguistics, in the LINDAT/CLARIN digital library ${ }^{8}$ [3]. This release retained the original Prague-style annotation before conversion to the UD standard.

\section{CONVERSION TO UNIVERSAL DEPENDENCIES}

The conversion of the annotation to the scheme defined in Universal Dependencies consists of two partially independent steps: 1 . converting the morphological tags to universal POS tags and features, and 2. converting the dependency relations.

The Interset Perl library ${ }^{9}$ [11] was used to convert the values of morphological categories to UD features; since the internal representation of Interset is defined as a kind of Interlingua for morphosyntactic tagsets, and because the features in UD are based directly on Interset, the conversion was rather straightforward. Most of the categories encoded in SNK tags were directly mappable to UD feature values, with the exception of paradigma (inflection class) for which there is no direct counterpart in UD.

\footnotetext{
${ }^{8}$ http://hdl.handle.net/11234/1-1822

${ }^{9}$ http://ufal.mff.cuni.cz/interset
} 
The situation is less straightforward with part-of-speech categories (the first character of SNK tags). UD guidelines define 17 universal part-of-speech tags (UPOS) that are rather coarse-grained but assumed to be sufficient for any natural language. If more fine-grained distinctions are needed, they should be encoded by additional features (UPOS tags are separate from features in UD).

\begin{tabular}{|l|l|l|l|}
\hline SNK & Description & UPOS & Features \\
\hline S & noun & NOUN, PROPN & \\
\hline A & adjective & ADJ & \\
\hline P & pronoun & PRON, DET & \\
\hline N & numeral & NUM & \\
\hline V & verb & VERB, AUX & \\
\hline G & participle & ADJ & VerbForm=Part \\
\hline D & adverb & ADV & \\
\hline E & preposition & ADP & \\
\hline O & conjunction & CCONJ, SCONJ & \\
\hline T & particle & PART & \\
\hline J & interjection & INTJ & \\
\hline R & reflexive pronoun & PRON & Reflex=Yes \\
\hline Y & conditional morpheme & AUX & Mood=Cnd \\
\hline W & abbreviation & X & Abbr=Yes \\
\hline Z & punctuation & PUNCT & \\
\hline Q & unidentifiable & X & Hyph=Yes \\
\hline$\#$ & non-word element & X & \\
\hline$\%$ & citation in foreign language & X & Foreign=Yes \\
\hline 0 & digit & NUM & NumForm=Digit \\
\hline
\end{tabular}

Tab. 3. Correspondence between SNK POS tags and UPOS (universal part-of-speech tags)

Table 3 shows the correspondences between SNK POS tags and UPOS. Certain ambiguities are relatively easy to solve - for example, common and proper nouns are distinguished by subsequent characters in the SNK tag. Other ambiguities cannot be resolved by looking at tags alone, and they are addressed outside Interset, taking also the word and its lemma into account. Thus all pro-adjectives (pronouns inflecting and behaving like adjectives) are listed and re-tagged DET in UD (see [12] for a discussion of pronouns vs. determiners in Slavic languages). The feature PronType (pronominal type) is set for all pronouns, determiners and pronominal adverbs. Ordinal and multiplicative numerals are distinguished from cardinals by the feature NumType and by changing their tag to ADJ or ADV (the NUM tag is reserved for definite cardinal numbers). Similarly, a word list is used to distinguish coordinating and subordinating conjunctions.

Another change involves polarity of verbs. In SNK, the negative forms with the prefix ne- are treated as derivational morphology: they are not encoded in the morphological tags and negative verbs have different lemmas than their affirmative counterparts (e.g. obvinit' "to accuse" - neobvinit" "not to accuse"). The Slovak UD data, on the other hand, use the affirmative lemma for both forms and set the Polarity 
feature to either "Pos" or "Neg". This is in line with the UD guidelines and improves parallelism to the Czech treebanks in UD. Note that negative verbs can be recognized using simple regular expressions, but one must watch for a few exceptions where an affirmative verb starts with ne- (nechat', nechávat', nenávidiet').

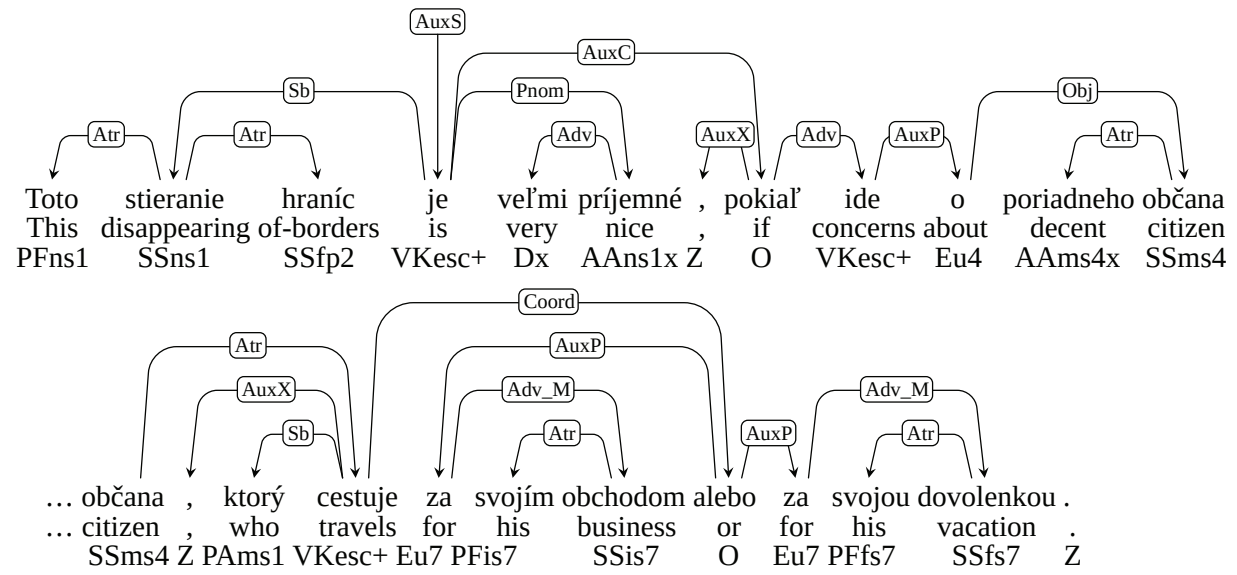

Fig. 1. Example of an original Prague-style dependency tree

Further part-of-speech adjustments occur during the transformation of the syntactic structure. For instance, the verb byt "to be" usually functions as an auxiliary verb or a copula. If it is found in one of these functions, its tag is changed from VERB to AUX.

Conversion of syntactic annotation is illustrated in Figures 1 and 2. Besides simple relabeling of dependency relations (see also Table 4), it involves several structural transformations:

- Copula verb heads the non-verbal predicate in the Prague style while the nonverbal predicate is the head in UD: je prijemne "is nice".

- In Prague, preposition is plugged as a connector between its noun and the parent of the prepositional phrase. In UD, prepositions are leaves attached to their nouns: o občana "about citizen", za obchodom "for business".

- In Prague, subordinating conjunction is plugged as a connector between the predicate of the subordinate clause and its parent. In UD, subordinating conjunctions are leaves attached to the predicates: pokial' ide "if it concerns".

- In Prague, coordination is headed by a conjunction or punctuation symbol; the child nodes are marked as either members of coordination (" M" attached to afun) or modifiers shared by the members (no suffix). In UD, coordination is headed by the first conjunct (member) and the subsequent conjuncts are attached to it. Shared modifiers cannot be distinguished from private modifiers of the first conjunct. 


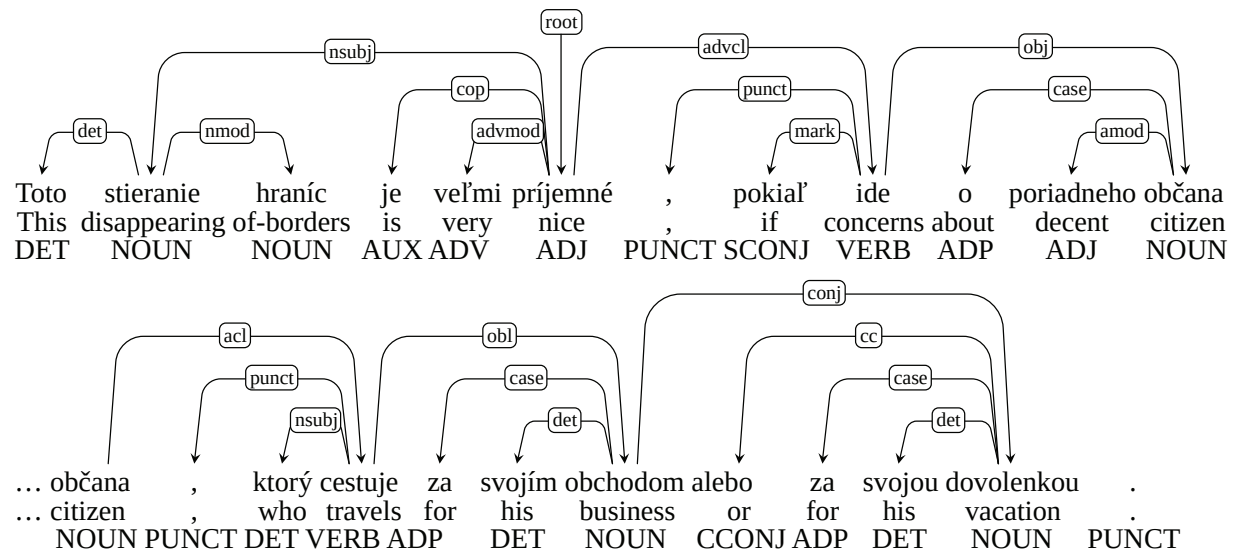

Fig. 2. The tree from Figure 1 converted to Universal Dependencies

\begin{tabular}{|l|l|l|}
\hline SNK & Description & UD \\
\hline Adv & adverbial modifier & obl, advmod, advcl \\
\hline Apos & apposition & appos*, punct* \\
\hline Atr & attribute & amod, det, nummod, nmod, flat, acl \\
\hline Atv & verbal attribute & acl \\
\hline AtvV & verbal attribute & xcomp \\
\hline AuxC & subordinating conjunction & mark* \\
\hline AuxG & non-comma punctuation & punct \\
\hline AuxK & sentence-final punctuation & punct* \\
\hline AuxO & semantically redundant & discourse \\
\hline AuxP & preposition & case* \\
\hline AuxR & reflexive passive & expl:pass \\
\hline AuxT & inherently reflexive verbs & expl:pv \\
\hline AuxV & auxiliary verb & aux, aux:pass \\
\hline AuxX & comma & punct \\
\hline AuxY & extra conjunction & cc, mark \\
\hline AuxZ & emphasizer & advmod:emph \\
\hline Coord & coordination head & cc*, conj*, punct* \\
\hline ExD & ex-dependent (ellipsis) & vocative, advcl, orphan*, dep \\
\hline Obj & object & obj, iobj, ccomp, xcomp \\
\hline Pnom & nominal predicate & cop* \\
\hline Pred & main predicate & root, parataxis \\
\hline Sb & subject & nsubj, nsubj:pass, csubj, csubj:pass \\
\hline
\end{tabular}

Tab. 4. Correspondence between SNK (Prague style) and UD dependency relations. The correspondences marked * are indirect: a structural transformation is necessary when the source relation occurs; the target relation may appear in the resulting structure but it will hold between a different pair of nodes.

The conversion procedure is not trivial because sometimes the rules outlined above interact. Notice how coordination is combined with prepositional phrases in our example - in the Prague style, the real type of the relation between cestuje and 
za obchodom, "Adv", is revealed two levels lower than in the UD tree. Fortunately, there was already software for conversion between the Prague style and UD. The publicly available Treex package ${ }^{10}$ [7] in the configuration described in [13] (with some extensions) was reused to convert the Slovak treebank.

The Slovak UD treebank first appeared in the Universal Dependencies release 1.4 in November 2016. In order to facilitate reproducibility of machine learning experiments, the dataset was split to training, development and test section, respectively (Table 5).

\begin{tabular}{|l|l|l|}
\hline Section & Sentences & Tokens \\
\hline Training & 8,483 & 80,575 \\
\hline Development & 1,060 & 12,440 \\
\hline Test & 1,061 & 13,028 \\
\hline
\end{tabular}

Tab. 5. The official split of the Slovak UD treebank into training, development and test data

The second edition that included Slovak, UD 2.0 in March 2017, followed the updated version of the UD guidelines, v2. (All examples in the present article also relate to the v2 guidelines.) The data split was the same as in UD 1.4 but the test sets were released separately after the CoNLL 2017 shared task in dependency parsing.

\section{USAGE AND RELATED WORK}

The mere fact that the treebank is available under a free license is very important for the Slovak language in the field of natural language processing. Being a part of a large collection like Universal Dependencies is a bonus that significantly increases visibility of the corpus. According to the statistics published by LINDAT/CLARIN, there have been 79 unique downloads of the Prague-style release of the Slovak Dependency Treebank, 2,592 unique downloads of UD 1.4 and 1985 unique downloads of UD 2.0 (as of July 19, 2017).

The treebank can be searched on-line in the PML-TQ search engine maintained by the Charles University in Prague ${ }^{11}$ and in the SETS engine at the University of Turku. $^{12}$

Soon after its first release, the treebank was picked (together with Czech, Slovenian, Croatian, Danish, Swedish and Norwegian) by the organizers of the VarDial 2017 shared task in parsing closely related languages [10]. A much larger shared task was organized as part of the CoNLL 2017 conference $^{13}$ [14]. The topic was end-to-end parsing from raw text, via automatic tokenization, sentence segmentation, lemmatization and morphological tagging to universal dependencies. The task set the new state of the art in dependency parsing for 45 languages, including Slovak. Baseline models were produced by the UDPipe system ${ }^{14}$ [9]; this

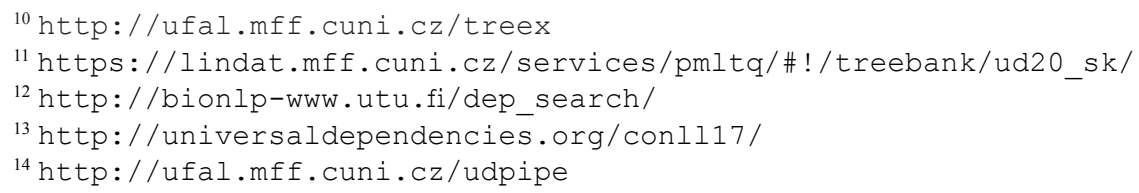


parser is open-source and available together with the pre-trained language models. Twenty of the systems competing in the shared task managed to surpass the baseline result; some of them are freely available, too.

The best results for Slovak were achieved by the team from Stanford: $83.86 \%$ content-word labeled attachment score (CLAS), 86.04\% labeled attachment score (LAS) and 89.58\% unlabeled attachment score (UAS). The parser was processing raw text (that is, it could not access gold-standard sentence segmentation, tokenization and morphology). All models were only trained on the training portion of the Slovak UD treebank. However, since much larger tagged data are available in the Slovak National Corpus, there is room for a significant boost of the tagging accuracy, which in turn may improve parsing results (but note that some parsers do not need morphology on input).

In connection to the shared task, large web corpora have been collected from CommonCrawl and Wikipedia for all the languages. The data have been automatically segmented, lemmatized, tagged and parsed by UDPipe, so there is now also a parsebank of Slovak comprising over 59 million sentences (811 million words) [4]. The first 2 million words have been indexed and made searchable through the SETS engine in Turku.

\section{CONCLUSION AND OUTLOOK}

We have presented the first public release of the Slovak Dependency Treebank and its automatic conversion to Universal Dependencies using rule-based heuristics and correspondence tables. We have shown that the release practically immediately put Slovak in several interesting NLP research projects where multilingual approaches are studied.

The current version contains only sentences with $100 \%$ inter-annotator agreement. This temporary measure ensures quality of the syntactic annotation but it also means that the released dataset is relatively small and unbalanced. It may not be easy to find human resources and funds to complete the disagreement resolution in the near future; however, we believe that there is room for checking additional sentences semi-automatically.

There are 7,564 sentences (95K tokens) where there was just one disagreement point between the annotators. Some of the mismatches mentioned in Section 2 may not be important for UD conversion or may be easily fixable. [8] notice that one of the most frequently confused pair of relations is AuxT (reflexive pronoun of an inherently reflexive verb) and AuxR (reflexive pronoun used to form reflexive passive). Both of them would be subtypes of expl in UD. Another frequent mismatch is AuxX vs. Coord. More research would be needed but if it signals inconsistent encoding of coordination, it could be normalized automatically.

Observations of this kind will hopefully help to speed up the completion of the remaining annotated data. Once all of them are added, the future releases of the Slovak Dependency Treebank will be four times bigger than the current one. 


\section{ACKNOWLEDGEMENTS}

The work was supported by the grant 15-10472S of the Czech Science Foundation. We have been using language resources and tools developed, stored and distributed by the LINDAT/CLARIN project of the Ministry of Education, Youth and Sports of the Czech Republic (project LM2015071).

The author wishes to thank Mária Šimková, Katarína Gajdošová and the entire team of the Ludovít Štúr Institute of Linguistics for their work on the treebank and for the permission to release it.

\section{References}

[1] Bejček, E., Hajičová, E., Hajič, J., Jínová, P., Kettnerová, V., Mikulová, M., Mírovský, J., Nedoluzhko, A., Panevová, J., Poláková, L., Ševčíková, M., Štěpánek, J., and Zikánová, Š. (2013). Prague dependency treebank 3.0. LINDAT/CLARIN digital library at the Institute of Formal and Applied Linguistics, Charles University. Accessible at: http://hdl.handle. net/11858/00-097C-0000-0023-1AAF-3.

[2] Boguslavsky, I., Iomdin, L., Petrochenkov, V., Sizov, V., and Tsinman, L. (2013). A case of hybrid parsing: Rules refined by empirical and corpus statistics. In Gerdes, K., Hajičová, E., and Wanner, L., editors, Computational Dependency Theory, volume 258, pages 226-240, IOS Press, Amsterdam, Netherlands.

[3] Gajdošová, K., Šimková, M., et al. (2016). Slovak dependency treebank. LINDAT/CLARIN digital library at the Institute of Formal and Applied Linguistics, Charles University. Accessible at: http: //hdl.handle.net/11234/1-1822.

[4] Ginter, F., Hajič, J., Luotolahti, J., Straka, M., and Zeman, D. (2017). CoNLL 2017 shared task automatically annotated raw texts and word embeddings. LINDAT/CLARIN digital library at the Institute of Formal and Applied Linguistics, Charles University. Accessible at: http://hdl . handle.net/11234/1-1989.

[5] McDonald, R., Nivre, J., Quirmbach-Brundage, Y., Goldberg, Y., Das, D., Ganchev, K., Hall, K., Petrov, S., Zhang, H., Täckström, O., Bedini, C., Bertomeu Castelló, N., and Lee, J. (2013). Universal dependency annotation for multilingual parsing. In Proceedings of the $51^{\text {st }}$ Annual Meeting of the Association for Computational Linguistics, pages 92-97, Sofija, Bulgaria.

[6] Nivre, J., de Marneffe, M.-C., Ginter, F., Goldberg, Y., Hajič, J., Manning, C., McDonald, R., Petrov, S., Pyysalo, S., Silveira, N., Tsarfaty, R., and Zeman, D. (2016). Universal Dependencies v1: A multilingual treebank collection. In Proceedings of the $10^{\text {th }}$ International Conference on Language Resources and Evaluation (LREC 2016), pages 1659-1666, Portorož, Slovenia.

[7] Popel, M. and Žabokrtský, Z. (2010). TectoMT: modular NLP framework. In Advances in Natural Language Processing: 7th International Conference on NLP, IceTAL 2010, pages 293-304, Springer, Berlin Heidelberg, Germany.

[8] Šimková, M. and Garabík, R. (2006). Синтаксическая разметка в Словацком национальном корпусе. In Труды международной конференции Корпусная лингвистика - 2006, pages 389 394, St. Petersburg University Press, Russia.

[9] Straka, M., Hajič, J., and Straková, J. (2016). UDPipe: trainable pipeline for processing CoNLL-U files performing tokenization, morphological analysis, POS tagging and parsing. In Proceedings of the $10^{\text {th }}$ International Conference on Language Resources and Evaluation (LREC 2016), pages 4290-4297, ELRA, Portorož, Slovenia.

[10] Zampieri, M., Malmasi, S., Ljubešić, N., Nakov, P., Ali, A., Tiedemann, J., Scherrer, Y., and Aepli, N. (2017). Findings of the VarDial evaluation campaign 2017. In Proceedings of the Fourth Workshop on NLP for Similar Languages, Varieties and Dialects (VarDial), Valencia, Spain.

[11] Zeman, D. (2008). Reusable tagset conversion using tagset drivers. In Calzolari, N., Choukri, K., Maegaard, B., Mariani, J., Odijk, J., Piperidis, S., and Tapias, D., editors, Proceedings of the $6^{\text {th }}$ 
International Language Resources and Evaluation Conference, LREC 2008, pages 28-30, Marrakech, Morocco.

[12] Zeman, D. (2015). Slavic languages in Universal Dependencies. In Natural Language Processing, Corpus Linguistics, E-learning, pages 151-163, RAM-Verlag, Lüdenscheid, Germany.

[13] Zeman, D., Dušek, O., Mareček, D., Popel, M., Ramasamy, L., Štěpánek, J., Žabokrtský, Z., and Hajič, J. (2014). HamleDT: Harmonized multi-language dependency treebank. Language Resources and Evaluation, 48(4):601-637.

[14] Zeman, D., Popel, M., Straka, M., Hajič, J., Nivre, J., Ginter, F., Luotolahti, J., Pyysalo, S., Petrov, S., Potthast, M., Tyers, F., Badmaeva, E., Gökırmak, M., Nedoluzhko, A., Cinková, S., Hajič jr., J., Hlaváčová, J., Kettnerová, V., Urešová, Z., Kanerva, J., Ojala, S., Missilä, A., Manning, C., Schuster, S., Reddy, S., Taji, D., Habash, N., Leung, H., de Marneffe, M.-C., Sanguinetti, M., Simi, M., Kanayama, H., de Paiva, V., Droganova, K., Martínez Alonso, H., Çöltekin, Ç., Sulubacak, U., Uszkoreit, H., Macketanz, V., Burchardt, A., Harris, K., Marheinecke, K., Rehm, G., Kayadelen, T., Attia, M., Elkahky, A., Yu, Z., Pitler, E., Lertpradit, S., Mandl, M., Kirchner, J., Fernandez Alcalde, H., Strnadová, J., Banerjee, E., Manurung, R., Stella, A., Shimada, A., Kwak, S., Mendonça, G., Lando, T., Nitisaroj, R., and Li, J. (2017). CoNLL 2017 shared task: Multilingual parsing from raw text to Universal Dependencies. In Proceedings of the CoNLL 2017 Shared Task: Multilingual Parsing from Raw Text to Universal Dependencies. Association for Computational Linguistics. Vancouver, Canada. 